\title{
The RecQ DNA helicases: Jacks-of-all-trades or master-tradesmen?
}

\author{
Neil Hunter ${ }^{1}$ \\ ${ }^{1}$ Sections of Microbiology and Molecular \& Cellular Biology, University of California Davis, One Shields Ave., Davis, CA 95616, \\ USA.nhunter@ucdavis.edu \\ Cell Research (2008) 18:328-330. doi: 10.1038/cr.2008.33; published online 3 March 2008
}

Homologous recombination occurs when a damaged chromosome uses an intact homologous chromosome as a template for its repair. The main steps of recombination are most readily illustrated for the repair of a DNA doublestrand-break (DSB). First, DSB-ends are processed to form single-stranded tails, which assemble into nucleoprotein complexes comprising an oligomeric filament of a RecA-family protein $(\operatorname{Rad} 51$ in eukaryotes) and associated factors. Rad51 filaments catalyze homologous pairing and strand-exchange between a DSB-end and a double-stranded template to form a joint molecule intermediate. This structure allows de novo priming of DNA synthesis to restore sequences that were lost or damaged at the site of the original lesion. Recombination also underpins chromosome replication by facilitating the repair of broken replication forks. In this case, joint molecule formation allows replication to reinitiate. At the final step of recombination, strand-exchange "Holliday" junctions that connect the involved chromosomes are resolved so that segregation can ensue. Joint molecule resolution can occur with one of two outcomes: a crossover, in which chromosome arms are exchanged; or a noncrossover without exchange.

Aberrant recombination causes chromosomal alterations that may activate oncogenes, cause loss-of-heterozygos- ity $(\mathrm{LOH})$ for tumor-suppressor genes and ultimately lead to transformation and tumorigenesis. It follows that recombination is regulated at multiple levels. Perhaps most important is to limit the use of recombination to the repair of lesions that cannot be appropriately repaired by other mechanisms. In addition, fidelity is maintained by confining recombination to the $\mathrm{S} / \mathrm{G} 2$ stages of the cell cycle, when sister-chromatid templates are available. Additional processes function to bias recombination to occur between identical, allelic sequences on the sister-chromatids and to be resolved with a noncrossover outcome. Recombination between parental homologs is less desirable because it can cause local sequence homozygosis, if the outcome is noncrossover, or $\mathrm{LOH}$ for all sequences distal to the site of recombination if the outcome is crossover. Moreover, interhomolog crossing-over in $\mathrm{S} / \mathrm{G} 2$ cells forms mitotic bivalents, which may be prone to segregation errors. Recombination becomes extremely hazardous when it occurs between non-allelic (ectopic) sequences; in such cases, crossing-over will lead to gross chromosomal changes such as translocations, inversions, duplications and deletions. The potential risks of recombination are compounded when non-identical (termed homeologous) templates are utilized.

The importance of regulating homol- ogous recombination is highlighted by a set of heritable cancer-prone diseases caused by mutations in recombination enzymes. Amongst these are the RecQ helicase disorders Bloom's syndrome, Werner's syndrome and RothmundThomson syndrome caused respectively by mutations in the related $B L M, W R N$ and RECQL4 genes [1]. The RecQ DNA helicases are an SF-II helicase subfamily with structurally and functionally conserved members in most bacteria and in all eukaryotes. They catalyze the prototypical helicase reaction of ATPdriven separation of complementary DNA strands, but are characterized by a preference for binding and unwinding branched DNA structures that arise during replication and recombination [2].

The role of RecQ enzymes in regulating homologous recombination is exemplified by the archetypal Bloom's helicase, BLM. Mitotic crossing-over, revealed as cytologically detectable exchanges between both sister and non-sister chromatids, is increased by an order of magnitude in cells lacking BLM. A mechanism for this anticrossover function was revealed by in vitro studies showing that BLM and associated factors can resolve joint molecule intermediates to specifically promote the noncrossover outcome of recombination. Most notably, BLM together with the type-I topoisomerase, TOPIII $\alpha$ and the binding/specificity factor, BLAP75, 
uniquely catalyze the "dissolution" of double-Holliday junction $(\mathrm{dHJ})$ joint molecule intermediates $[3,4]$.

Consistent with the distinct pathologies of Werner's and Rothmund-Thomson syndromes, cells lacking WRN and RECQL4 do not share the hypercrossover phenotype of BLM mutants, and both WRN and RECQL4 enzymes have distinct biochemical properties. WRN comprises both RecQ helicase and exonuclease activities and plays an important role in telomere stability, probably by regulating telomere-telomere recombination [1]. RECQL4 is essential for DNA replication in Xenopus oocyte extracts, where it helps recruit or stabilize polymerases at replication origins [5]. While RECQL4 is not essential for DNA replication in human cells it is required to arrest cells in S-phase in response to genotoxic stress [6]. Surprisingly, recombinant RECQL4 lacks detectable helicase activity despite being a DNA-dependent ATPase [1]. Perhaps the biological activity of RECQL4 is achieved by translocating along DNA without strand-separation, or by simply binding to a specific DNA structure such as a replication fork. By analogy to the sole budding yeast RecQ helicase, Sgs1, RECQL4 may act to stabilize polymerases at stalled replication forks [7].

Vertebrate genomes contain two additional RecQ proteins, RECQL and RECQL5, which have yet to be associated with heritable human diseases. RECQL is the closest in structure to $E$. coli RecQ, lacking the $\mathrm{N}$ - and $\mathrm{C}$-terminal domains characteristic of BLM, WRN and RECQL4. The hyperrecombination and chromosomal instability phenotypes of human and mouse cells lacking RECQL suggest functional overlap with BLM [8]. Also analogous to BLM, RECQL interacts with several components of the DNA mismatch repair system and can disrupt joint molecules [2]. However, RECQL is distinct from BLM in that it cannot catalyze the dHJ dissolution reaction, despite being able to interact with TOPIII $\alpha$. Yeast Sgs1 functions with the DNA mismatchrepair machinery to suppress recombination between homeologous sequences [9]. Perhaps the most important function of RECQL is to suppress this risky type of recombination in vertebrates.

Hu et al. [11] recently provided new insights into the biological functions of RECQL5. Previous work by the Luo group showed that Recql5 $5^{--}$mutant mouse cells, like $\mathrm{Blm}^{-/}$cells, have elevated frequencies of crossing-over [10]. They now show that Recql $5^{--}$knockout mice are also highly cancer prone [11]. The functions of RECQL5 and BLM are clearly not redundant, however, as epistasis analysis demonstrates that Recql5 and $B l m$ function nonredundantly to suppress crossing-over [10]. Striking differences between RECQL5- and BLM-deficient cells provide further evidence for distinct cellular functions of these two helicases.

First, LOH is not elevated in Recql5 $5^{-1}$ ES cells, whereas LOH increases by at least 10 -fold in $\mathrm{Blm}^{-/}$cells. This result is perplexing given the increased crossing-over detected in Recql5 $5^{-/}$cells and raises the question of why Recql $5^{--}$cells are cancer prone? A possible explanation is suggested by the response of Recql5 ${ }^{-/}$cells to camptothecin (CPT), an inhibitor of topoisomease I that induces fork stalling and breakage; a $\sim 10$-fold induction of chromosomal aberrations is detected, including chromosome breaks and fusions, multiradial structures (indicative of interchromosomal crossingover) and complex rearrangements. A similar effect of CPT on chromosomal stability was not detected in $\mathrm{Blm}^{-/}$cells. Thus, the cancer susceptibility of Recql5 $5^{--}$mice may be primarily due to oncogene activation caused by chromosomal rearrangements. This idea raises the testable prediction that the tumors that develop in Recql5 $5^{-/}$and $\mathrm{Blm}^{--}$mice will have distinct underlying etiologies. Furthermore, Recql5 $5^{--}$and $\mathrm{Blm}^{--}$mice might be expected to develop distinct types of tumor. The prominence of lung adenocarcinomas in Recql5 $5^{-/}$mice hints that this could be the case.

Consistent with the inference that RECQL5 regulates recombination, the frequency and lifespan of Rad51 immunostaining foci is increased in Recql5 $5^{-\leftarrow}$ cells, indicating an abnormal accumulation and/or persistence of recombination intermediates in these cells. Revealingly, the fraction of DSBs that are repaired via homologous recombination is increased in Recql5 $5^{-/}$cells. Taken together, these data suggest that RECQL5 acts to prevent "unscheduled" recombination. Moreover, the additional lesions that are channeled into the recombination pathway in Recql5 $5^{-/}$cells appear to be repaired abnormally.

The relationship between murine Recql5 and Blm parallels that of the Srs2 and Sgs1 helicases, two key regulators of recombination in budding yeast. Sgs1 appears to be structurally and functionally analogous to BLM. The unrelated Srs2, an SF-I superfamily helicase, prevents unscheduled recombination at replication forks by displacing Rad51 from single-stranded DNA [12]. This antirecombination effect is mediated by an interaction between Srs2 and the replicative clamp, PCNA. Extending the analogy with Srs2, Hu et al. [11] showed that human RECQL5 similarly inhibits Rad51 from catalyzing joint molecule formation by displacing it from singlestranded DNA. Moreover, RECQL5 also interacts with PCNA and localizes to replication forks. Is RECQL5 the vertebrate ortholog of yeast Srs2? This seems unlikely as vertebrate genomes encode an additional helicase, FBH1, which may be structurally and functionally more closely equivalent to $\mathrm{Srs} 2$ than is RECQL5 [13].

Accompanying the study of $\mathrm{Hu}$ et al. [11], an investigation by Bugreev et al. [15] demonstrated that human BLM is also capable of disrupting Rad51 filaments in vitro. Thus, at least three DNA helicases, BLM, RECQL5 and Srs2 (and presumably vertebrate FBH1) may negatively regulate recom- 
bination by disrupting Rad51 filament formation (note that RECQL and WRN cannot catalyze this reaction). Differences between the activities of these three helicases argue against simple redundancy. Srs2 can disrupt filaments comprised of ssDNA and either yeast Rad51, human Rad51, or even E. coli RecA indicating a general displacement activity. However, Srs 2 does not dissociate Rad51-coated strand-exchange products (joint molecules), in which the Rad51 is now bound to the double-stranded (ds) product of strandexchange. Consistently, Srs 2 cannot disrupt Rad51 filaments assembled onto linear dsDNA [14]. In contrast, human BLM can disrupt Rad51-coated joint molecules although it is unclear whether this activity involves direct dissociation of dsDNA-Rad51 complexes [15]. Also, BLM can only disrupt cognate filaments of human Rad51, suggesting the need for a specific BLM-Rad51 protein-protein interaction; filaments comprised of yeast $\operatorname{Rad} 51$ or human Dmc1, a $\operatorname{Rad} 51$ homolog, are not disrupted. Moreover, disruption by BLM only occurs when Rad51 filaments are in the inactive, ADP-bound state. Finally, experiments using ATPase defective Rad51 protein suggest that RECQL5 can, distinctively, displace active ATP-bound Rad51-filaments [11].

As the complexity of the RecQ helicases unfolds, what can we surmise about the relationships between the five vertebrate RecQ proteins? First, differences between their structures and biochemical activities, and the distinct phenotypes of human and mouse mutants argue against substantial redundancy. It is notable, however, that BLM appears to have some redundancy with most or all of the other RecQ homologs. We can suggest that the primordial eukaryotic RecQ helicase evolved the plethora of activities that can be demonstrated for human BLM and yeast Sgs1 proteins. From this "Jack-of-all-trades", via gene amplification, mutation and selection, specific activities may have been parceled-out to produce a set of "master-tradesmen", dedicated to one or a few activities. Ultimately, this division of RecQ labor may have enabled metazoans to exert finer spatial and temporal regulation over specific RecQ activities.

\section{References}

1 Hanada K, Hickson ID. Molecular genetics of RecQ helicase disorders. Cell Mol Life Sci 2007; 64:2306-2322.

2 Sharma S, Doherty KM, Brosh RM Jr. Mechanisms of RecQ helicases in pathways of DNA metabolism and maintenance of genomic stability. Biochem J 2006; 398:319-337.

3 Wu L, Bachrati CZ, Ou J, et al. BLAP75/ RMI1 promotes the BLM-dependent dissolution of homologous recombination intermediates. Proc Natl Acad Sci USA 2006; 103:4068-4073.

4 Raynard S, Bussen W, Sung P. A double Holliday junction dissolvasome comprising BLM, topoisomerase IIIalpha, and BLAP75. J Biol Chem 2006; 281:13861-13864.

5 Sangrithi MN, Bernal JA, Madine M, et al. Initiation of DNA replication requires the RECQL4 protein mutated in Rothmund-Thomson syndrome. Cell 2005; 121:887-898.

6 Park SJ, Lee YJ, Beck BD, Lee SH. A positive involvement of RecQL4 in UVinduced S-phase arrest. DNA Cell Biol 2006; 25:696-703.

7 Cobb JA, Bjergbaek L, Shimada K, Frei C, Gasser SM. DNA polymerase stabilization at stalled replication forks requires Mec1 and the RecQ helicase Sgs1. EMBO J 2003; 22:4325-4336.

8 Sharma S, Brosh RM. Human RECQ1 is a DNA damage responsive protein required for genotoxic stress resistance and suppression of sister chromatid exchanges. PLoS ONE 2007; 2:e1297.

9 Spell RM, Jinks-Robertson S. Examination of the roles of Sgs1 and Srs2 helicases in the enforcement of recombination fidelity in Saccharomyces cerevisiae. Genetics 2004; 168:1855-1865.

$10 \mathrm{Hu}$ Y, Lu X, Barnes E, et al. Recq15 and Blm RecQ DNA helicases have nonredundant roles in suppressing crossovers. Mol Cell Biol 2005; 25:3431-3442.

$11 \mathrm{Hu}$ Y, Raynard S, Sehorn MG, et al. RECQL5/Recq15 helicase regulates homologous recombination and suppresses tumor formation via disruption of Rad51 presynaptic filaments. Genes Dev 2007; 21:3073-3084.

12 Branzei D, Foiani M. RecQ helicases queuing with Srs2 to disrupt Rad51 filaments and suppress recombination. Genes Dev 2007; 21:3019-3026.

13 Kohzaki M, Hatanaka A, Sonoda E, et al. Cooperative roles of vertebrate Fbh1 and Blm DNA helicases in avoidance of crossovers during recombination initiated by replication fork collapse. $\mathrm{Mol}$ Cell Biol 2007; 27:2812-2820.

14 Veaute X, Jeusset J, Soustelle C, et al. The Srs2 helicase prevents recombination by disrupting Rad51 nucleoprotein filaments. Nature 2003; 423:309-312.

15 Bugreev DV, Yu X, Egelman EH, Mazin AV. Novel pro- and anti-recombination activities of the Bloom's syndrome helicase. Genes Dev 2007; 21:3085-3094. 